\title{
Fibrous cartilage of human menisci is less shock-absorbing and energy-dissipating than hyaline cartilage
}

\author{
Mario Gaugler • Dieter Wirz • Sarah Ronken • \\ Mirjam Hafner • Beat Göpfert • Niklaus F. Friederich • \\ Reinhard Elke
}

Received: 18 September 2013/ Accepted: 20 February 2014/Published online: 11 March 2014

(C) Springer-Verlag Berlin Heidelberg 2014

\begin{abstract}
Purpose To test meniscal mechanical properties such as the dynamic modulus of elasticity $E^{*}$ and the loss angle $\delta$ at two loading frequencies $\omega$ at different locations of the menisci and compare it to $E^{*}$ and $\delta$ of hyaline cartilage in indentation mode with spherical indenters.

Methods On nine pairs of human menisci, the dynamic $E^{*}$-modulus and loss angle $\delta$ (as a measure of the energy dissipation) were determined. The measurements were performed at two different strain rates (slow sinusoidal and fast single impact) to show the strain rate dependence of the material. The measurements were compared to previous similar measurements with the same equipment on human hyaline cartilage.

Results The resultant $E^{*}$ at fast indentation (median $1.16 \mathrm{MPa}$ ) was significantly higher, and the loss angle was significantly lower (median $10.2^{\circ}$ ) compared to slowloading mode's $E^{*}$ and $\delta$ (median $0.18 \mathrm{MPa}$ and $16.9^{\circ}$, respectively). Further, significant differences for different locations are shown. On the medial meniscus, the anterior horn shows the highest resultant dynamic modulus.

Conclusion In dynamic measurements with a spherical indenter, the menisci are much softer and less energy-
\end{abstract}

M. Gaugler · D. Wirz $(\bowtie) \cdot$ S. Ronken · M. Hafner

B. Göpfert · N. F. Friederich

Center of Biomechanics and Calorimetry Basel, University of

Basel, Klingelbergstrasse 50-70, 4056 Basel, Switzerland

e-mail: dieter.wirz@unibas.ch

M. Hafner

Health Division, Bern University of Applied Sciences,

Murtenstrasse 10, 3008 Bern, Switzerland

R. Elke

Orthomerian, Gotthelfstrasse 105, 4054 Basel, Switzerland dissipating than hyaline cartilage. Further, the menisci are stiffer and less energy-dissipating in the middle, intermediate part compared to the meniscal base. In compression, the energy dissipation of meniscus cartilage plays a minor role compared to hyaline cartilage. At high impacts, energy dissipation is less than on low impacts, similar to cartilage.

Keywords Meniscus cartilage - Fibrocartilage $\cdot$ Hyaline cartilage $\cdot$ Mechanical properties $\cdot$ In vitro study

\section{Introduction}

The menisci play crucial mechanical functions in the knee. As already pointed out by Fithian [7], they distribute stress, absorb shocks and enhance congruity between femoral condyle and tibial plateau. The meniscus function is load transmission by enlarging the tibio-femoral contact area and decreasing the contact stress during load and impact [2]. Besides this congruency enhancement and the rotational stabilization [10], the menisci are considered as shock absorbers [1]. This shock absorption is claimed to be caused by the morphological structure and the water permeability of fibrous cartilage [7].

Shock-absorbing materials are usually modelled as a combination of dashpots and springs, and therefore, the dynamic modulus of elasticity $E^{*}$, the aggregate modulus, can be separated into a real part $E^{\prime}$, representing linear elasticity, and a virtual part $E^{\prime \prime}$, representing the energy dissipation, where

$E^{* 2}=E^{\prime 2}+E^{\prime \prime 2}$

and

$\tan (\delta)=E^{\prime} / E^{\prime \prime}$ 
$\delta$, the loss angle, is a measure for the part of energy that is dissipated during a loading cycle. As previously reported for hyaline cartilage [14], all these material constants are dependent from the loading frequency $\omega$ or strain rate: With an increase in the loading frequency, $E^{*}$ increases and $\delta$ decreases in viscoelastic materials.

The mechanical properties of the menisci have been determined mostly in tension [8,9], and only some few studies in compression [4, 16] have been performed. Sweigart et al. [16] examined the creep of medial menisci of different species in indentation. But to our knowledge, no dynamic measurements have been performed in indentation mode. A recently published paper by Sandman et al. [15] compared meniscus stiffness, residual force and relative sample compression comparing human and mammalian menisci and Actifit $^{\circledR}$ made of polyurethane and Menaflex ${ }^{\circledR}$ made of collagen showing much less viscoelastic behaviour than fibre cartilage.

The goal of the presented paper was to determine the dynamic modulus of elasticity $E^{*}$ and the loss angle $\delta$ at two loading frequency $\omega$ at different locations. Not the bulk properties of the menisci were determined, but the material properties of the fibrous cartilage of human menisci. Further, the results will be compared to $E^{*}$ and $\delta$ of hyaline cartilage. To our knowledge, this is the first study measuring dynamically at two different strain rates in indentation mode the aggregate Modulus $E^{*}$. The E-modulus was separated in an elastic part $E^{\prime}$ and a viscous part $E^{\prime \prime}$ as a measure of the energy dissipation. These results might have impact on the design und clinical use of artificial meniscus implants.

\section{Materials and methods}

Nine pairs of menisci were harvested from fresh frozen and thawed knee joints from human donors: age 62.7 years (54-78), six right and three left knees, four male and five female, body mass index 24.8 (17-31). The menisci were dissected on the whole and embedded in PMMA [Poly (methyl methacrylate), SCS Beracryl biphasic D28, W. Troller Kunststoffe AG, Jegenstorf, Switzerland] with the upper side up. The menisci were zoned into anterior, corpus, and posterior segments, respectively, outer, centre and inner measuring points, which gives a total of 18 locations per meniscus (Fig. 1). During the measurements, the specimens were kept moistened with phosphate-buffered saline.

The measurements were performed with a single-impact micro-indentation device (fast indentation, FI) and with slow-sinusoidal indentation (SS) @ $0.1 \mathrm{~Hz}$ on a MTS Synergie 100 (MTS Systems Corp., Eden Prairie MN,

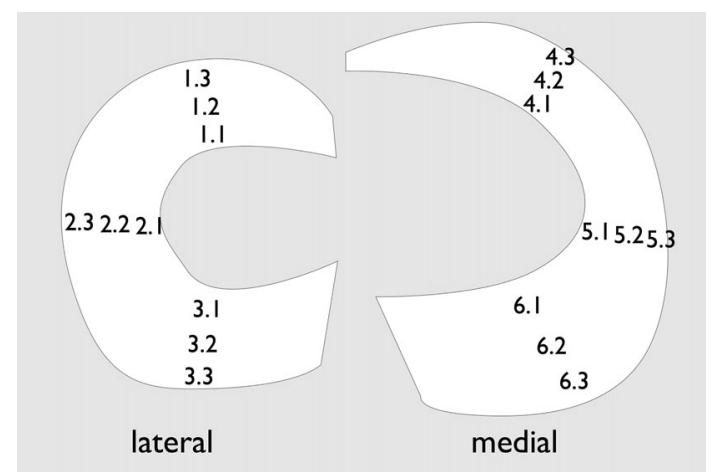

Fig. 1 Measurement points on the menisci: one anterior horn medial, two corpus medial, three posterior horn medial and 4-6 lateral meniscus, the numbers after the dot indicate one central, two intermediate and two outer rim, basis

USA) testing device, both measurement set-ups have been described in detail by Ronken et al. [14].

In similar experiments with fresh porcine knee cartilage, repeated FI tests were found to be non-destructive [19]. At each location, 11 repetitions at an interval of approximately $30 \mathrm{~s}$ were performed. The inner and outer measurement points where not accessible for the FI measurement device because of the closed concept of the FI. It is not possible to determine within $<2 \mathrm{~mm}$ where the indenter hits the surface. Therefore, only the middle measuring points i.e. measurement points 1.2, 2.2, 3.2, 4.2, 5.2 and 6.2 were examined. For the slow indentations (SS) at each location, three repetitions at an interval of approximately $30 \mathrm{~s}$ were performed.

In both indentation modes maximum forces and maximum displacement were not occurring at the same time (Fig. 2). The phase angle $\delta$ can be extracted directly from this phase shift.

Aggregate modulus and loss angle were calculated with the mathematical model of Vriend and Kren [18]. This model assumes infinite thickness of the specimen. Therefore, the indentation depth had to be kept below $10 \%$ of the specimen thickness [5]. Following this $10 \%$ rule of Bückle, the higher stiffness of the supporting material is not affecting the results. The aggregate modulus and loss angles were determined for every single impact, and then, the median was calculated to get one standard set of values for each measuring point.

The menisci originate from knees, which were approved for mechanical research by the local ethical committee (Ethikkommission beider Basel, EK 52/11).

Statistical analysis

Two-sample $t$ test and power analysis for unequal sample sizes were carried out; $p$ values $<0.05$ were regarded to be 

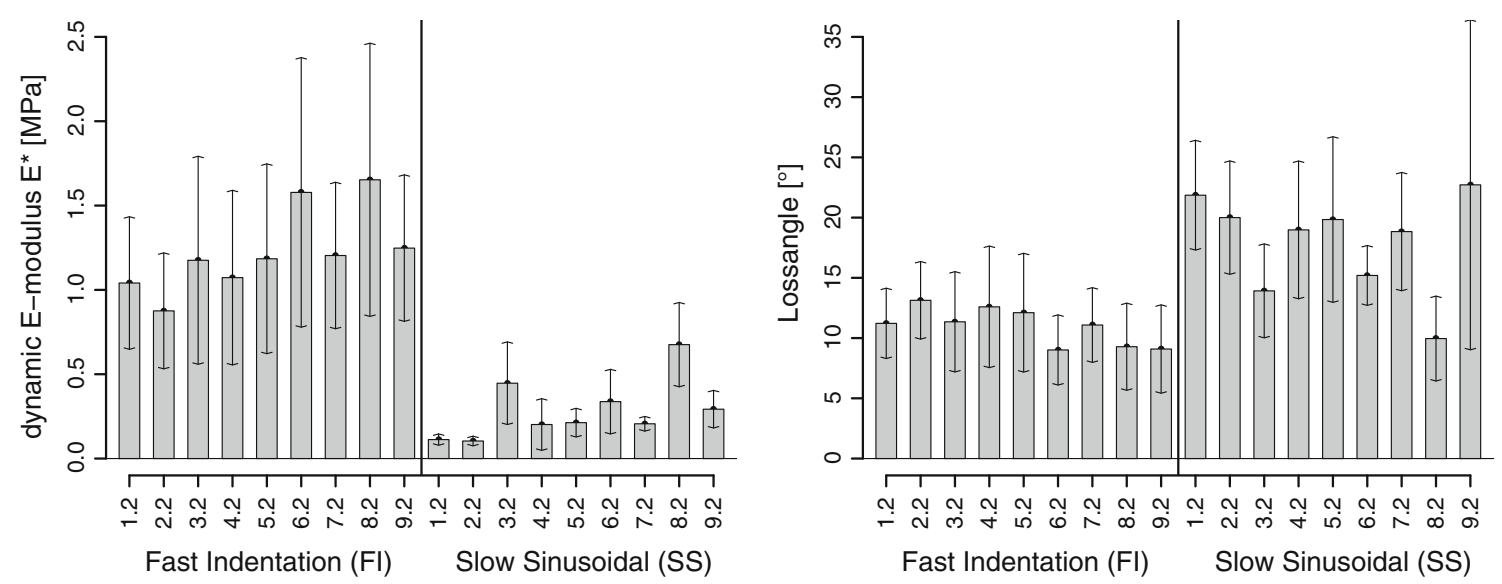

Fig. 2 Illustration of the time shift between displacement and force (SS, specimen 5, measuring point 1.1), the vertical lines indicate the time shift of the 2nd cycle

statistically significant. A power analysis was performed to determine the probability of a type II error, and the limit for power was set to 0.8. ANOVA-tests were carried out to compare the inner, middle and outer measurement points on the menisci. Mathematical modelling, statistical analysis and plots are performed in R [R Core Team (2012). R: A language and environment for statistical computing. R Foundation for Statistical Computing, Vienna, Austria. ISBN 3-900051-07-0, URL http://www.R-project.org/].

\section{Results}

The results of all the measurements are presented in Fig. 3.

In viscoelastic tissues and materials, a faster impact generate a higher $E^{*}$ and lower $\delta$, which means the material gets stiffer and dissipates less energy. At any location, $E^{*}$ were significantly higher and the loss angles significantly lower at high strain rate and vice versa.

The resultant aggregate modulus at fast indentation with a median of $1.2 \pm 0.6 \mathrm{MPa}$ (median and median absolute deviation) was significantly higher, and the median of the loss angle was significantly lower $10.2 \pm 3.3^{\circ}$, compared to slow-loading modes $E^{*}=0.18 \pm 0.13 \mathrm{MPa}$, respectively, $\delta=16.9 \pm 6.9^{\circ}$. Two-sample $t$ test showed significant differences comparing the loss angles and aggregate moduli at the middle locations of both loading modes.

On the medial meniscus, the anterior horn has the highest resultant aggregate modulus $E^{*}=1.7 \pm 0.9 \mathrm{MPa}$, and the posterior horn, $E^{*}=1.1 \pm 0.4 \mathrm{MPa}$, is stiffer at location 2.2 with an aggregate modulus of $0.9 \pm 0.4 \mathrm{MPa}$. Contrary, on the lateral meniscus, stiffness increases toward posterior. At high strain rate, the posterior horn, $E^{*}=1.3 \pm 0.8 \mathrm{MPa}$, is the stiffest location on the lateral meniscus. The anterior segment has an aggregate modulus of $E^{*}=1.1 \pm 0.5 \mathrm{MPa}$ and is less stiff than the corpus with $E^{*}=1.2 \pm 0.4 \mathrm{MPa}$. Unlike aggregate modulus, no significant differences for loss angles at FI for the different middle locations (n.s.) could be found. Figure 4 on the right-hand side illustrates that under fast loading mode, the loss angle values for all locations result in equal ranges; hence, all locations dissipated similar amounts of energy $\left(\delta=10.2 \pm 3.3^{\circ}\right)$.

The stiffness pattern in anterior-posterior alignment at slow strain rate is the same as at fast indentation. The anterior horn of the medial meniscus $\left(E^{*}=\right.$ $0.21 \pm 0.17 \mathrm{MPa})$ and the posterior horn of the lateral meniscus $\left(E^{*}=0.27 \pm 0.23 \mathrm{MPa}\right)$ are the stiffest locations. In inner-outer orientation, the $E^{*}$ value decreases by more than $40 \%$ as the following results show, where the inner rims are the stiffest with $E^{*}=0.24 \pm 0.22 \mathrm{MPa}$, and the middle locations $E^{*}=0.22 \pm 0.16 \mathrm{MPa}$ are stiffer than the outer locations $E^{*}=0.14 \pm 0.07 \mathrm{MPa}$. The median of the $E^{*}$ values of the basis is significantly lower than that of the central, middle and intermediate measuring
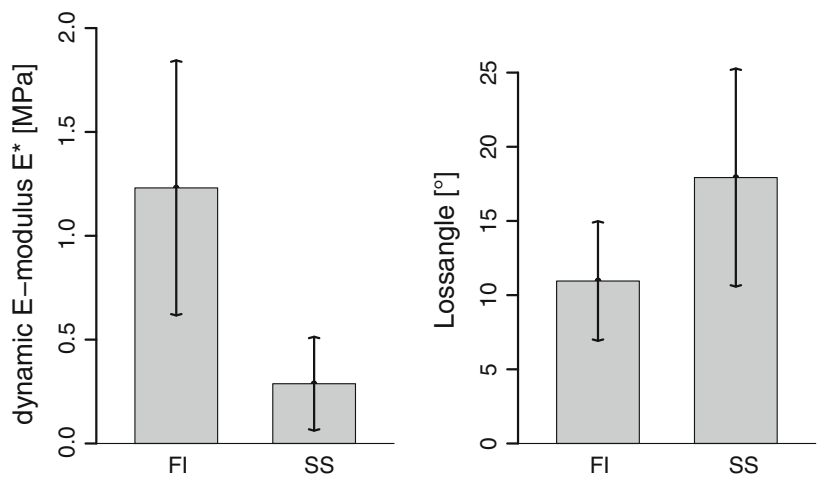

Fig. 3 Mean and standard deviation dynamic E-modulus and loss angle of all nine specimen at all intermediate measurement points at fast indentation (FI) ad slow-sinusoidal indentation (SS) 

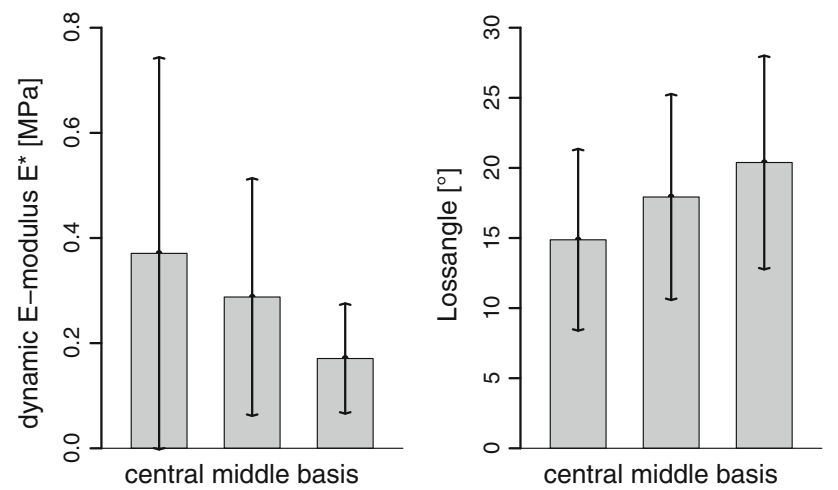

Fig. 4 Mean and standard deviation of dynamic E-modulus (left) and loss angle (right) of all specimens at slow indentation (SS) comparing inner rim (central), middle part and close to the meniscus basis
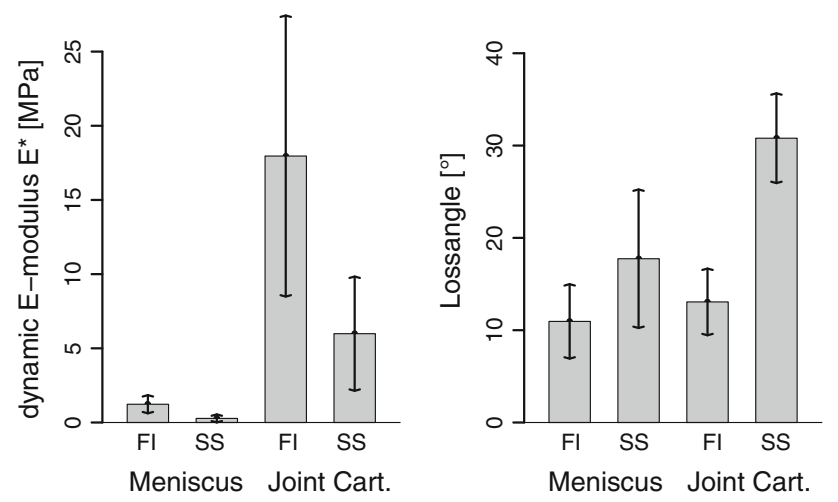

Fig. 5 Comparison (mean and standard deviation) of previous measurements of hyaline joint cartilage slow-sinusoidal (SS) and fast indentation (FI) [14] versus meniscus cartilage at slow-sinusoidal (SS) and at fast indentation (FI)

points (Fig. 5). The inner rims of both medial and lateral menisci dissipate significantly less energy $\delta=14 \pm 6^{\circ}$ than the middle $\delta=16 \pm 6^{\circ}$ and the outer rims $\delta=19 \pm 7^{\circ}$, which have the highest loss angles (Fig. 4).

Finally, the results were compared to the already published data from Ronken et al. [14]. In this study by Ronken et al., the cartilage stiffness was measured without detachment from the underlying bone on small bone-cartilage cylinders. Using the same testing devices, it was a suitable reference to compare aggregate modulus and loss angles, see Fig. 5.

In both loading modes, $E^{*}$ of hyaline cartilage was about $10 \times$ higher than meniscus. Loss angles of cartilage and meniscus at fast indentation were in the same range, whereas in slow-sinusoidal mode, meniscus dissipates approximately only half the energy of articular cartilage. All these differences are significant; however, the probability of a type II error for the comparison of the loss angles in fast indentation are $50 \%$.

\section{Discussion}

The most important finding of the present study was the result that the stiffness and the energy dissipation (at least at low strain rates) are much less for menisci than for joint cartilage. Further, the stiffness and the energy dissipation of meniscus cartilage varied significantly considering test mode and location. The stiffness pattern of both loading modes investigated in this work fit the trend of Sweigart et al. [16] with the anterior horn to be the stiffest location on the medial meniscus and verify that the indentation methods FI and SS used in this work are an accurate method for measuring the mechanical properties of meniscus. The results acquired of the medial meniscus at slow indentation are in range of those examined by Sweigart et al. using a creep indentation. The stiffness scheme is adapted to the load-transmission forces according to Pena et al. [13]. In areas where maximum contact pressure occurs (medial meniscus posterior region and lateral meniscus anterior region), the tissue is designed to transmit less energy and thereby reduce peak pressure. As expected, significant stiffening, due to pressurization of intestinal fluid, occurred at increased loading rates, which is consonant with its function of load transmission and match the results of Baro et al. [4]. As discussed by Park et al. [12], higher strain rates in viscoelastic materials lead to higher $E^{*}$ and lower $\delta$. At FI, no significant differences for the different locations were found; thus, under fast loadings, all locations dissipate equal amounts of energy. In anteriorposterior and inner-outer direction, differences were found at slow-sinusoidal indentation. The inner zones are significantly stiffer and have lower loss angles than the middle and outer locations. With its lower and different collagen composition [6] and higher proteoglycan content [11, 17], the inner rims resemble more to hyaline cartilage. These highly hydrated proteins might decrease water content, resulting in a higher aggregate modulus.

A limiting factor of this study is that some of the menisci were injured or altered; hence, a big dispersion is seen between the specimen's material properties. However, specimen three had a severe chondrocalcinosis which did not seem to influence the mechanical properties at fastindentation, and in slow mode, a higher median aggregate modulus and lower loss angle was noticeable. Specimen four's and six's injuries are not giving the impression to affect its overall material properties. Specimen eight looked worn out and dehydrated, resulting in a stiffer $E^{*}$ for both loading modes and a lower loss angle at SS. Due to limited specimen quantity, these differences were not evaluated statistically.

To compare the mechanical properties of hyaline cartilage to meniscus, a study of Ronken et al. [14] on comparison of human and porcine cartilage was considered. 
The result that meniscus cartilage is much softer than hyaline cartilage is not new, but we were able to confirm these results with dynamic measurements. However, the loss angles of meniscus cartilage are lower than the loss angles of hyaline cartilage, at least in indentation measurements. This difference is much more distinct at low loading rates than at high loading rates. The low loading rates (SS) compare to standing around and moving the weight from one leg to the other, whereas high loading rates (FI) occur during the heal strike in fast walking or running.

As mentioned above, loss angle is a measure for energy dissipation or in other words, of the shock-absorbing capabilities of a material. The menisci are commonly regarded as shock absorbers; however, Andrews et al. [3] claim in their 2011 review article "that the menisci may play no significant role in shock absorption". The present paper shows that meniscus cartilage has some shockabsorbing capabilities, mainly at low speed impacts, but less than hyaline cartilage. With the formula (2) (tan $\left.(\delta)=E^{\prime} / E^{\prime \prime}\right)$, we can calculate that only a small part (around $18 \%$ ) of the energy is dissipated. The dumping properties of meniscal fibre cartilage seem not to play the important role usually assigned to the menisci. Although Sandman et al. [15] claim that artificial menisci are inferior viscoelastic, polyurethane polymers therefore can be considered as possible replacement material.

The menisci are only tested in indentation. This is the main limitation in the presented work. It cannot be concluded that the energy dissipation is similar in tensionand probably the main part of the shock absorbance occurs in tension. As already mentioned, the meniscus functions are congruency enhancement and rotational stabilization, but at least in compression the energy dissipation plays a minor role.

\section{Conclusions}

The aggregate modulus of meniscus cartilage varied significantly considering test mode and location. The slowloading mode seems to be more sensitive identifying discrepancies in loss angles as a function of location. The strain rate dependency underlines the need of multi-mode test for evaluating function related properties.

At low loading rates i.e. sinusoidal cycles at $0.1 \mathrm{~Hz}$, the menisci show much lower loss angles than hyaline cartilage. The energy applied on menisci is much less dissipated at low impacts, such as standing or slowly walking, than in hyaline cartilage. The energy dissipation decreases at high impacts as it is the case for all poro-viscoelastic materials, and it is close to that of hyaline cartilage.
Acknowledgments This study was supported by the Merian Iselin Clinic for Orthopaedics and Surgery, Föhrenstrasse 2, Basel, Switzerland.

\section{References}

1. Aagaard H, Verdonk R (1999) Function of the normal meniscus and consequences of meniscal resection. Scand J Med Sci Sports 9:134-140

2. Ahmed AM (1995) The load-bearing role of the knee menisci. In: Arnoczky SP, Jackson DW (eds) Mow VC. Knee meniscus Basic Clin Found Raven Press, New York, pp 59-73

3. Andrews S, Shrive N, Ronsky J (2011) The shocking truth about meniscus. J Biomech 44:2737-2740

4. Baro VJ, Bonnevie ED, Lai X, Price C, Burris DL, Wang L (2012) Functional characterization of normal and degraded bovine meniscus: rate-dependent indentation and friction studies. Bone 51:232-240

5. Bückle H (1973) The science of hardness testing and its research applications. In: Westbrook W, Conrad H (eds) American Society for Metals. Metals Park, OH, pp 453-494

6. Cheung HS (1987) Distribution of type I, II, III and V in the pepsin solubilized collagens in bovine menisci. Connect Tissue Res Informa UK Ltd UK 16:343-356

7. Fithian DC, Kelly MA, Mow VC (1990) Material properties and structure-function relationships in the menisci. Clin Orthop Relat Res 252:19-31

8. Goertzen DJ, Budney DR, Cinats JG (1997) Methodology and apparatus to determine material properties of the knee joint meniscus. Med Eng Phys 19:412-419

9. LeRoux MA, Setton LA (2002) Experimental and biphasic FEM determinations of the material properties and hydraulic permeability of the meniscus in tension. J Biomech Eng 124:315-321

10. Mariani PP (2011) Posterior horn instability of the medial meniscus a sign of posterior meniscotibial ligament insufficiency. Knee Surg Sport Traumatol Arthrosc 19:1148-1153

11. Nakano T, Dodd CM, Scott PG (1997) Glycosaminoglycans and proteoglycans from different zones of the porcine knee meniscus. J Orthop Res 15:213-220

12. Park S, Hung CT, Ateshian GA (2004) Mechanical response of bovine articular cartilage under dynamic unconfined compression loading at physiological stress levels. Osteoarthr Cartil 12:65-73

13. Pena E, Calvo B, Martinez MA, Palanca D, Doblaré M (2005) Finite element analysis of the effect of meniscal tears and meniscectomies on human knee biomechanics. Clin Biomech 20:498-507

14. Ronken S, Arnold MP, Ardura García H, Jeger A, Daniels AU, Wirz D (2012) A comparison of healthy human and swine articular cartilage dynamic indentation mechanics. Biomech Model Mechanobiol 11:631-639

15. Sandmann GH, Adamczyk C, Garcia EG, Doebele S, Buettner A, Milz S, Imhoff AB, Vogt S, Burgkart R, Tischer T (2013) Biomechanical comparison of menisci from different species and artificial constructs. BMC Musculoskelet Disord BioMed Central Ltd 14:324

16. Sweigart MA, Zhu CF, Burt DM, DeHoll PD, Agrawal CM, Clanton TO, Athanasiou KA (2004) Intraspecies and interspecies comparison of the compressive properties of the medial meniscus. Ann Biomed Eng 32:1569-1579

17. Tanaka T, Fujii K, Kumagae Y (1999) Comparison of biochemical characteristics of cultured fibrochondrocytes isolated from the inner and outer regions of human meniscus. Knee Surg Sport Traumatol Arthrosc 7:75-80 
18. Vriend NM, Kren AP (2004) Determination of the viscoelastic properties of elastomeric materials by the dynamic indentation method. Polym Test 23:369-375
19. Wirz D, Kohler C, Keller K, Göpfert B, Hudetz D, Daniels AU (2008) Dynamic stiffness of articular cartilage by single impact micro-indentation (SIMI). J Biomech 41:S172 